\title{
Florian Franken
}

Überreden und Überzeugen

\section{Eine Verhältnisbestimmung aus der Perspektive des späten Wittgenstein}

\begin{abstract}
The word 'persuasion' can be used in two different ways. It can either implicate a process of changing someone's belief or action without any argumentative justification or, to the contrary, indicate that the changes are indeed a result of argumentative discourse. These two different uses are part of a conceptual development in the history of philosophy. Nowadays they are often placed in contrast to each other, whereby persuasion in philosophy as a non-argumentative act is taken as doubtful and even unsound. In the following paper I argue that the two types of processes should not be conceived of as incompatible, taking some notes from Wittgenstein's On Certainty as an argumentative source. I will show that persuading as a rational practice is based on the possibility of persuasion as a process of inculcation. These processes can help illuminate the notions of good grounds, reasons and persuasion. In contrast to the common perspective, I will show that they are actually the normal case regarding interaction between humans and thus fundamentally provide the possibility of an argumentative practice of giving and accepting reasons. I conclude that the primacy of argumentative persuasion in relation to inculcation needs to be reversed and the common understanding of human beings as genuine rational acting beings needs to be questioned.
\end{abstract}

Keywords: Wittgenstein, persuasion, reason, justification, good grounds, rationality, belief, certainty, argumentative discourse, disagreement.

DOI 10.1515/dzph-2015-0003

Spätestens seit Platon wird der Unterschied zwischen zwei kommunikativen Vorgehensweisen philosophisch reflektiert, nämlich der zwischen dem Vorgang

Dr. des. Florian Franken: Ludwig-Maximilians-Universität, Fakultät für Philosophie, Wissenschaftstheorie und Religionswissenschaft, Geschwister-Scholl-Platz 1, 80539 München; florian. franken@gmx.de 
der Überredung und dem Vorgang des Überzeugens. ${ }^{1}$ Bevor die Überredung durch die Sophistik aufgrund ihrer technischen Anwendung in der Rhetorik eine praktische Aufwertung erfuhr, waren die Vorsokratiker noch davon überzeugt, dass die Überredung der Wahrheit folge und zur Gewissheit des Seienden führe. ${ }^{2}$ Doch bereits Platons Sokrates unterscheidet die Überredung als einen Prozess, dessen Ziel es ist, statt mit richtigem Wissen zu belehren, zweifelhaften Glauben zu erregen. ${ }^{3}$ Er hält die sophistische Rhetorik keineswegs für eine Kunst, sondern für eine moralisch zweifelhafte, von einer „dreisten Seele“ vollzogenen „Schmeichelei““. Diesem unschönen Vorgehen stellt Platon die philosophische Rhetorik gegenüber, einem Überreden mittels Dialektik, das durch argumentative

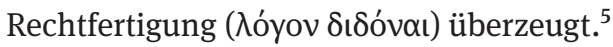

Die Gegenüberstellung von ,überreden` und ,überzeugen` erfolgt ab dem 18. Jahrhundert in aufkommender Häufigkeit. Zu dieser Zeit setzt sich in Verbindung mit dem Begriff der Überzeugung ebenfalls der Begriff des Fürwahrhaltens durch, der sich auf die Gültigkeit einer Erkenntnis bezieht. ${ }^{6}$ Kant beispielsweise unterscheidet zwischen zwei Weisen des Fürwahrhaltens, das sowohl von objektiven Gründen als auch von subjektiven Ursachen des urteilenden Subjekts abhängig ist. Eine Überzeugung liegt dann vor, wenn ihr Anspruch auf intersubjektive Gültigkeit durch objektiv hinreichende Gründe eingelöst werden kann. Währenddessen resultiert die Überredung daraus, dass der Grund lediglich für objektiv gehalten wird und daher „bloßer Schein“ ist. Sie besitzt daher bloß private Gültigkeit und ist folglich im Bereich der subjektiv und objektiv unzugänglichen Meinung angesiedelt. ${ }^{7}$

Aufgrund der Tatsache, dass der gegenwärtig etablierte Wissensbegriff die Notwendigkeit der Rechtfertigung impliziert, so scheint es, genießt der Begriff der Überzeugung ein weitaus höheres Ansehen und wird philosophisch für wichtiger erachtet als der Begriff der Überredung. Darüber hinaus ist unser gegenwärtiges Rationalitätsverständnis und unser damit verbundenes Selbstverständnis als rationale Wesen in weiten Teilen der Tatsache einer tiefsitzenden Verbindung zu

1 Interessanterweise macht das englische Wort „persuasion“ (griech. $\pi \varepsilon^{\prime} \theta \varepsilon \imath v$; lat. persuadere) keinen signifikanten Unterschied zwischen diesen beiden Vorgehensweisen.

2 Vgl. Parmenides, VS 28, in: Diels/Kranz (1903), 113.

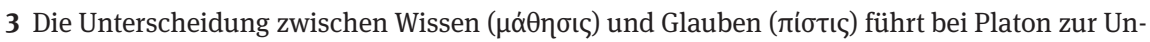
terscheidung zweier Arten der Überredung: Neben der „glaubenmachenden Überredung“ in der Rhetorik ist die Rede von einer „belehrenden Überredung“ (vgl. Gorg. 454d-455a). Letztere ist mit dem gleichzusetzen, was Platon unter dem Vorgang des Überzeugens versteht.

4 Vgl. Gorg. 463a.

5 Vgl. Phaidr. 271c-d, 273 d-e; Prot. 336b-c.

6 Vgl. Diemer (1972), 1149.

7 Vgl. Kant (1983), A 820/B 848 ff. 
einer Praxis des Gebens und Annehmens von Gründen geschuldet, die im Dienst der Rechtfertigung von individuellen Überzeugungen und Handlungen steht. Obwohl der Begriff des Überzeugens im Sinne eines argumentativen Diskurses historisch gesehen im Hinblick auf die Rechtfertigung von Wissensansprüchen und die Erklärung von Handlungen eine außerordentlich wichtige Bedeutung zu haben scheint, möchte ich im Folgenden indes behaupten, dass der Begriff der Überredung, unter dem ich der Einfachheit halber einen Vorgang der Meinungsoder Verhaltensänderung jenseits einer argumentativ-diskursiven Praxis verstanden wissen möchte, grundlegend für ein Verständnis in Bezug darauf ist, was es heißt, im Besitz von Gründen zu sein, bzw. die Fähigkeit zu besitzen, Gründe zu geben und anzunehmen. Ohne die Möglichkeit des grundlegenden Vorgangs einer Überredung, so werde ich argumentieren, ist die Bedeutung eines auf Gründen basierenden Vorgangs des Überzeugens nicht verständlich zu machen. Ich verfolge damit das Ziel, den unter Philosophen von Beginn an in Verruf geratenen Überredungsbegriff in einer bestimmten Hinsicht $\mathrm{zu}$ rehabilitieren und seine grundlegend positive Bedeutung in den Vordergrund zu stellen.

Ich werde mich zu diesem Zweck auf einige Überlegungen des späten Wittgenstein beziehen, insbesondere auf Notizen, die in Über Gewissheit zusammengefasst worden sind, und sie zum Gegenstand einer ausführlichen Analyse machen. Auf diesem Wege werde ich zeigen, dass der Vorgang des Überzeugens den Spezialfall einer propositionalen Übereinkunft im Rahmen eines menschlichen sozialen Verhaltens darstellt. Die Präsuppositionen dieses Spezialfalls sind indes in einer Situation verankert, die als Vorgang der Überredung interpretiert werden muss. Letzterer basiert, wie sich zeigen wird, auf einer grundlegenden Dynamik innerhalb eines Systems menschlicher Praktiken. Obwohl der Vorgang der Überredung selbst nicht Teil des argumentativen Diskurses ist, muss er dennoch so verstanden werden, dass er die Möglichkeit zu dessen Bestehen bereitet. Vor diesem Hintergrund soll also dafür argumentiert werden, dass die Übereinkunft, die durch den Vorgang des Überzeugens hergestellt wird, nicht originär durch argumentative Rechtfertigung erfolgt, sondern tatsächlich in einer Übereinkunft eingeübter sozialer Praktiken begründet ist. Sie stellt die Bedingung für die Möglichkeit des Gebens und Annehmens von Gründen dar und liefert somit die Präsuppositionen für das Erreichen eines Einverständnisses in den Überzeugungen durch Übereinkunft.

Um diese Behauptungen zu erläutern, lege ich als Ausgangspunkt die folgenden Passagen aus den Notizen Wittgensteins in Über Gewissheit zugrunde:

[608] Ist es falsch, dass ich mich in meinem Handeln nach dem Satze der Physik richte? Soll ich sagen, ich habe keinen guten Grund dazu? Ist [es] nicht eben das, was wir einen „guten Grund" nennen? 
[609] Angenommen, wir träfen Leute, die das nicht als triftigen Grund betrachteten. Nun, wie stellen wir uns das vor? Sie befragen statt des Physikers etwa ein Orakel. (Und wir halten sie darum für primitiv.) Ist es falsch, dass sie ein Orakel befragen und sich nach ihm richten? - Wenn wir dies „falsch“ nennen, gehen wir nicht schon von unserm Sprachspiel aus und bekämpfen das ihre?

[610] Und haben wir recht oder unrecht darin, dass wir's bekämpfen? Man wird freilich unser Vorgehen mit allerlei Schlagworten (slogans) aufstützen.

[611] Wo sich wirklich zwei Prinzipien treffen, die sich nicht miteinander aussöhnen, da erklärt jeder den Andern für einen Narren und Ketzer.

[612] Ich sagte, ich würde den Andern `bekämpfen`, - aber würde ich ihm denn nicht Gründe geben? Doch; aber wie weit reichen die? Am Ende der Gründe steht die Überredung. (Denke daran, was geschieht, wenn Missionare die Eingeborenen bekehren. $)^{8}$

Im Zentrum dieser Passage steht die Vorstellung einer tendenziell kämpferischen Auseinandersetzung zwischen zwei Gruppen von Menschen, die sich hinsichtlich ihrer normativen, handlungsleitenden Überzeugungen unterscheiden. Wittgenstein geht davon aus, dass das Aufeinandertreffen der beiden verschiedenen Gruppen in der Lage ist, einen Konflikt auszulösen. Um die Struktur dieses Konfliktes und seine Bedingungen in den Blick zu bekommen, werde ich entlang der zu analysierenden Passage drei weitreichende Fragen diskutieren, denen sich die folgenden Abschnitte unterordnen lassen und anhand derer ich meine Behauptungen argumentativ unterfüttern möchte: (1) Was sind ,gute Gründe‘? (2) Was sind ,überzeugende Gründe‘? Und (3) was heißt es, jemanden zu überzeugen? Diese drei Fragen scheinen mir eng miteinander verbunden zu sein. Ich werde sie einzeln untersuchen und dabei versuchen, die Ergebnisse in einen geeigneten Zusammenhang zueinander zu stellen.

\section{1}

In der Notiz ÜG 608 präsentiert Wittgenstein einen, wie es auf den ersten Blick scheinen mag, plausiblen Kandidaten im Hinblick darauf, was wir gemeinhin als einen ,guten Grund' bezeichnen. Man kann sich vorstellen, dass gute Gründe, mit denen Personen ihre Handlungen rechtfertigen, durch Sätze der Physik zur Verfügung gestellt werden. In Bezug auf Handlungen scheinen solche Sätze eine normative Bedeutung zu implizieren. Beispielsweise ist der Satz „Wasser siedet bei

8 Vgl. dazu auch Wittgenstein (1984b), 92 (im Folgenden zitiert als ÜG). Hervorhebungen im Original. 
$100{ }^{\circ} \mathrm{C}^{\prime}$ dazu in der Lage, die Handlung einer Person anzuleiten, die die Absicht hat, Salz von Salzwasser zu trennen. ${ }^{9}$ Dass eine Person eine Flamme entzündet und das Salzwasser erhitzt, um im Anschluss das übergebliebene Salz zu entnehmen, kann dadurch erklärt werden, dass die Person von der objektiven Gültigkeit des Satzes überzeugt ist. Gemeinsam mit der Absicht, Salz zu gewinnen, bildet die Überzeugung den Grund, der die Handlung der Person erklärt. ${ }^{10}$

Insofern sich die Überzeugungen einer Person auf die Mittel zum Erreichen eines Zieles oder Zweckes beziehen, ist leicht einzusehen, dass sie zum Bestehen von Gründen beitragen, die für die Ausführung einer Handlung angeführt werden können. In welcher Beziehung Mittel und Zwecke zueinander stehen, scheint durch Regeln festgelegt $\mathrm{zu}$ sein, die in entsprechenden Handlungen ihren Ausdruck finden, und dem Muster ,Wenn du X möchtest und du weißt, dass A zu X führt, dann tue A“ folgen. Zweckrationale Handlungen lassen sich folglich durch Regeln begründen, die ihrerseits Überzeugungen dazu voraussetzen, in welchem von der Regel ausgedrückten Verhältnis Mittel und Zweck zueinander stehen. ${ }^{11}$ Durch solche Regeln lassen sich Handlungen zwar rational begründen, aber damit ist nicht notwendig vorausgesetzt, dass die in diesem Zuge angegebenen Gründe auch als gute Gründe gelten können. ${ }^{12}$ Die sich anschließende Frage lautet demzufolge: Auf welche Weise kann eine Handlung, die sich zweckrational begründen lässt, durch gute Gründe gerechtfertigt

\footnotetext{
9 Inwiefern ein solcher Satz handlungsleitend ist, lässt sich klassischerweise mit Hilfe eines praktischen Syllogismus erläutern, in dessen Rahmen er gemeinsam mit der Absicht die beiden Prämissen darstellt, aus denen die Handlung folgt: $\left(\mathrm{P}_{1}\right) \mathrm{X}$ hat die Absicht Salz aus siedendem Salzwasser zu extrahieren; $\left(\mathrm{P}_{2}\right) \mathrm{X}$ hat die Überzeugung, dass Wasser bei $100{ }^{\circ} \mathrm{C}$ siedet; $(\mathrm{K}) \mathrm{X}$ erhitzt Salzwasser auf $100^{\circ} \mathrm{C}$.

10 Hempel und Oppenheim haben versucht, Handlungen in Form eines deduktiv-nomologischen Modells zu erklären, indem sie die Überzeugung als eine gesetzesmäßige Aussage interpretierten (Hempel/Oppenheim 1948). Dieses Modell ist von Davidson (1980) vor dem Hintergrund seiner Idee eines anomalen Monismus weiterentwickelt worden.

11 Anhänger einer Diskurstheorie gehen bezugnehmend auf die Sprechakttheorie und seit einiger Zeit mit Bezug auf die normative Pragmatik Brandoms davon aus, dass der „Raum der Gründe“ (Sellars, Brandom) mit dem Rückgriff auf formale Regeln zu rekonstruieren, d. h. explizit zu machen sei. Ich möchte auf das Missverständnis, das dieser Sichtweise zugrunde liegt, hier nicht erneut eingehen. Eine Reihe von Autoren haben in erhellender Weise darauf aufmerksam gemacht, dass Regeln weder in einem impliziten noch in einem expliziten Verhältnis zur Handlung stehen; vgl. z. B. Schneider (2002); Williams (2010); Kern (2010); Franken (2014). Sie weisen stattdessen auf die Identität von Regel und Praxis hin.

12 Forst meint, dass eine zweckrationale Begründung deshalb „normativ neutral“ sei (Forst 2007, 25). Diese Formulierung macht einen irreführenden Anschein, wenn man nicht berücksichtigt, dass Forst den Begriff normativer Gründe auf moralisch rechtfertigende Gründe reduziert. Ein solches Verständnis ist aber nicht zwingend, wie beispielsweise Korsgaard (1997) zeigt.
} 
werden? Die vorherige Annahme vorausgesetzt, dass Überzeugungen im Hinblick auf die Physik dazu beitragen, Handlungsgründe abgeben zu können, stellt sich die Frage, wodurch sich diese Überzeugungen als Bestandteil guter Gründe rechtfertigen lassen.

Ein vielversprechender Kandidat scheint in dieser Hinsicht die experimentelle Beobachtung zu sein. Betrachten wir erneut das vorherige Beispiel einer Handlung nun unter Aspekten im Rahmen eines Experiments. Die Person erhitzt Salzwasser über einer Flamme und misst dabei die Temperatur, um mittels einer Beobachtung den Zeitpunkt festzustellen, an dem das Wasser siedet. Als kochendes Wasser verdampft, schaut sie auf die Anzeige eines Thermometers und stellt fest, dass die Temperatur des Wassers $100{ }^{\circ} \mathrm{C}$ beträgt. Von dieser Beobachtung ausgehend, kann sie nun den Erfahrungssatz formulieren, dass Wasser bei $100{ }^{\circ} \mathrm{C} \mathrm{zu}$ sieden beginnt. Die Beobachtung scheint demzufolge ihre Überzeugung zu rechtfertigen und ihren Handlungsgrund dementsprechend als einen guten Grund auszuzeichnen. Die begründende Überzeugung der Person entpuppt sich auf diese Weise als ein Erfahrungssatz, der ihr Wissen durch empirische Beobachtung rechtfertigt. Dies klingt zunächst außerordentlich plausibel, doch ist es fraglich, ob dieser Zusammenhang auch einem zweiten Blick standhält.

Zunächst einmal muss eingeräumt werden, dass sich die Person während des Experimentes mit ihrer Beobachtung auch geirrt haben kann. Tatsächlich, so wollen wir einmal annehmen, hat das Thermometer nicht ,100 ${ }^{\circ} \mathrm{C}$, , sondern ,180 ${ }^{\circ} \mathrm{C}^{\prime}$ angezeigt. Gehen wir der Einfachheit halber davon aus, dass der Grund dafür in einem Defekt der Anzeige liege. Unter diesen Umständen ist die Behauptung, das Wasser siede bei $100{ }^{\circ} \mathrm{C}$, aufgrund der Beobachtung der fehlerhaften Anzeige falsch. Zweitens ist fraglich, auf welche Weise entschieden werden kann, dass die Beobachtung auf einer falschen Grundlage beruht. Wie kann hinreichend sichergestellt werden, dass das Thermometer richtig funktioniert?

Wenn wir sichergehen möchten, dass das erste Experiment entweder korrekt durchgeführt worden ist oder aber einen Irrtum in der Durchführung beinhaltet, scheint folglich das Experiment als solches zum Untersuchungsgegenstand gemacht werden zu müssen. Um die Korrektheit des ersten Experiments zu prüfen, könnte ein weiteres Thermometer zur Messung hinzugezogen werden. Als Konsequenz aus der Wiederholung des Experiments würde erwartet werden können, dass das Ergebnis des zweiten Thermometers sich von dem des ersten unterscheidet und nun die korrekte Messung anzeigt. Doch die vermeintliche Lösung des Problems entpuppt sich als ein schwerwiegendes Dilemma. (a) Nimmt man an, dass das zweite Thermometer das gleiche Ergebnis anzeigt, so kann die Person nicht sicher sein, dass die Anzeige dieses Mal den richtigen Wert wiedergibt. Es 
ist ja durchaus vorstellbar, dass auch die Anzeige dieses zweiten Thermometers defekt ist. In dieser Situation würde eine dritte Untersuchung der Untersuchung der Untersuchung - das scheint klar zu sein - erneut zu keinem befriedigenden Ausgang, sondern geradewegs in einen infiniten Regress hineinführen. (b) Wird dagegen angenommen, dass das zweite Thermometer ein anderes Ergebnis hervorbringt als die erste Messung, so ist unklar, was die Resultate des zweiten Thermometers über diejenigen des ersten aussagen können. Wenn beide Geräte in siedendem Wasser unterschiedliche Ergebnisse anzeigen, bleibt offen, welches Thermometer garantieren soll, dass das jeweils andere das korrekte oder aber ein inkorrektes Messergebnis anzeigt. Es bleibt daher unklar, wie und wodurch die Richtigkeit des Ergebnisses und damit die Wahrheit der Wissensbehauptung entschieden werden kann.

Das Dilemma zeigt, dass die Annahme, Wissensbehauptungen ließen sich durch die Erfahrung in Form experimenteller Beobachtung rechtfertigen, und die sich daraus ableitende Folgerung, Beobachtungssätze stellten gute Gründe dar, in tiefgreifende Schwierigkeiten führt. Deshalb ist die These äußerst zweifelhaft, dass handlungsanleitendes Wissen dadurch gerechtfertigt sei, dass es sich auf Beobachtungen stütze. Andererseits erreicht die extreme Gegenthese, dass Erfahrung überhaupt keine Rolle in Bezug auf Handlungsentscheidungen spiele, ebenfalls keine Zustimmung. Folglich besteht die Aufgabe darin, die Rolle von Erfahrung in Bezug auf die Bedeutung guter Gründe erneut zu überdenken.

Wenn behauptet wird, dass Erfahrung eine Wissensbehauptung rechtfertige oder dass Erfahrung in der Lage sei, gute Gründe zu liefern, dann wird ihr dabei eine gewisse Autorität in Bezug auf die Wahrheit einer Aussage zugesprochen. Man könnte dann ebenfalls geneigt sein zu behaupten, dass die Erfahrung uns als Betrachtern zeigt, oder, wie Wittgenstein es metaphorisch formuliert, uns „lehrt“ (ÜG 274), was wir wissen. Diesbezüglich stellt sich die Frage, wie es zu verstehen ist, dass Erfahrung uns etwas lehren kann. Bis hierhin ist deutlich geworden, dass einzelne Erfahrungssätze, die Wissensansprüche anhand von Experimenten bestätigen sollen, zur Beantwortung dieser Frage nicht in Betracht kommen.

Darüber hinaus ist zu berücksichtigen, dass einzelne Erfahrungssätze das Ergebnis einer Interpretation der gemachten Erfahrung sind. Um nicht einem permanenten Zweifel ausgesetzt zu sein, muss diese durch weitere, bereits vorhandene Überzeugungen gestützt werden. Daraus ist abzuleiten, dass der Erfolg eines auf Beobachtung basierenden Experimentes voraussetzt, dass die durch Interpretation gewonnenen empirischen Aussagen dadurch dem Zweifel entzogen werden, dass sie durch andere Aussagen gestützt werden, die bereits vom Zweifel ausgeschlossen sind. „Man kann nicht experimentieren“, bemerkt Witt- 
genstein, „wenn man nicht manches nicht bezweifelt. [...] Wenn ich experimentiere, so zweifle ich nicht an der Existenz des Apparates, den ich vor den Augen habe. Ich habe eine Menge Zweifel, aber nicht den.“ (ÜG 337, Hervorhebung im Original). Folglich ist mit der Aussage, die Erfahrung lehre uns etwas, bereits vorausgesetzt, dass sich dieser Vorgang nicht, wie es oberflächlich scheinen mag, durch die Äußerung eines einzelnen Erfahrungssatzes konstituiert, sondern vielmehr durch die Beteiligung einer „Menge zusammenhängender Sätze“ (ÜG 274), die Wittgenstein auch als ein „System“ von Erfahrungssätzen (ÜG 136) bezeichnet. In diesem System ist eine gewisse Menge von Sätzen notwendigerweise vom Zweifel ausgeschlossen. Unter dieser Bedingung wird das Hervorbringen eines epistemisch relevanten Urteils überhaupt erst möglich. ${ }^{13}$

Die Überlegungen in Bezug auf die Frage, in welcher Weise Erfahrung zu der Annahme von guten Gründen beiträgt, haben das Augenmerk auf Sätze gelenkt, die die Erscheinungsform empirischer Sätze aufweisen, ihnen gegenüber jedoch die Eigenschaft besitzen, nicht dem Zweifel ausgesetzt zu sein. Dieser Fokus scheint einen Zugang zum Verständnis der Bemerkung ÜG 608 zu öffnen, und zwar durch die Überlegung, dass Sätze der Physik die Form von Erfahrungssätzen aufweisen (vgl. ÜG 96), obwohl sie über jeden Zweifel erhaben sind. Gerade diese Eigenschaft ist es, die Wittgenstein heraushebt, wenn er betont, dass wir im Allgemeinen keine Gründe haben, die uns an den Sätzen der Physik zweifeln lassen. Es spricht nichts dagegen, dass wir uns in unserem Handeln an ihnen orientieren. In paradox anmutender Weise scheint es aber gerade die Grundlosigkeit zu sein, mit der wir uns praktisch nach den Sätzen der Physik richten, die deutlich macht, warum es „gute Gründe“ für ein solches Handeln gibt. Die Redeweise von der Grundlosigkeit als gutem Grund macht indes verständlich, dass gerade dann, wenn eine Handlung durch Gründe erklärt werden soll, bestimmte Überzeugungen von der Notwendigkeit der Begründung ausgeklammert sind und als begründet vorausgesetzt werden müssen, damit sich die Frage, die eine Begründung der Handlung verlangt, überhaupt sinnvoll stellen kann. Es leuchtet beispielsweise ein, dass eine Person, die sich gehend von einem Ort zum anderen bewegen möchte, einen auf Nachfrage anzugebenden Grund hat, ein Bein vor das andere zu setzen. Hat sie in dieser Absicht auch einen Grund, den Boden auf seine Tragfähigkeit hin zu untersuchen, auf dem sie geht? Unter normalen Umständen müsste diese Frage verneint werden. Trotzdem besitzt die Person,

13 Dazu auch ÜG 603: „Man lehrt mich, dass unter solchen Umständen dies geschieht. Man hat es herausgefunden, indem man den Versuch ein paarmal gemacht hat. Das alles würde uns freilich nichts beweisen, wenn nicht rund um diese Erfahrung andere lägen, die mit ihr ein System bilden." Hervorhebung im Original. 
gerade in Anbetracht der Grundlosigkeit, mit der sie von der Tragfähigkeit des Bodens unter ihren Füßen ausgeht, einen guten Grund, ein Bein vor das andere zu setzen. Andernfalls wäre sie gar nicht in der Lage den beabsichtigten Gang zu vollziehen.

Folglich stellt sich heraus, dass gerade in Zusammenhängen, in denen keine Gründe vorliegen, die geeignet wären gegen eine bestimmte Handlungsweise angeführt $\mathrm{zu}$ werden, dennoch offensichtlich gute Gründe vorliegen, in dieser bestimmten Weise zu handeln. Diese guten Gründe sind, wie deutlich geworden ist, nicht in der Weise konstituiert, dass sie Gegenstand des Austausches im Rahmen einer argumentativen Praxis sind. ${ }^{14}$ Ihre Wirksamkeit äußert sich offensichtlich in einer nichtpropositionalen Form. Für die Unterscheidung zwischen propositionalen und nichtpropositionalen Gründen lassen sich zwei schlagkräftige Argumente anführen, von denen wir eines bereits kennengelernt haben. Zum einen müssen wir in epistemologischer Hinsicht etwas Bestimmtes als bereits gewiss annehmen, um überhaupt etwas anderes bezweifeln zu können (vgl. ÜG 115). Dem lässt sich ein zweites Argument beistellen, das sich auf den Spracherwerb bezieht. Ich werde es im Folgenden erläutern.

Wie wir gesehen haben, stellt die Behauptung, dass eine Wissensbehauptung durch einzelne Erfahrungssätze gerechtfertigt werden könne, einen Irrtum dar. Dennoch betont Wittgenstein, dass der Erfahrung eine kardinale Rolle hinsichtlich des Anleitens und Rechtfertigens von Handlungen durch entsprechende Urteile zukomme. Die Art und Weise, wie wir zu solchen Urteilen gelangen, beruht jedoch nicht auf einer Erfahrung, deren Kern in der empirischen Beobachtung besteht. Ein auf empirische Beobachtung reduzierter Erfahrungsbegriff kann handlungsleitende Urteile nicht hinreichend erklären, weil empirische Urteile, wie gesagt, bereits das Ergebnis einer Interpretation der Erfahrung sind, deren Bedeutung in der Folge handlungsleitend ist (vgl. ÜG 145). Die Weise, in der die Interpretation der Erfahrung handlungsleitend ist, so möchte ich im Folgenden argumentieren, wird bestimmt durch ihre Einbindung in sozial geteilte Praktiken. ${ }^{15}$

Um diese Behauptung argumentativ zu unterfüttern, werde ich auf Wittgensteins bekanntes Beispiel einer primitiven Sprache zurückgreifen, das er im $\S 2$ seiner Philosophischen Untersuchungen anführt. Ein Bauender A hat seinen Gehilfen B darin eingeübt, ihm auf den Ruf „Platte“ eine Platte zu bringen. Immer dann, wenn A ruft, bringt B den Gegenstand. Bei der Betrachtung dieses Vorgangs ist zu berücksichtigen, dass die Interaktion der beiden Akteure enorm ein-

14 Kern (2010) spricht daher auch von einem „rationalen Handeln ohne Überlegen“.

15 Die Bedingung sozial geteilter Praktiken im Hinblick auf die Interpretation von Erfahrung stellt insbesondere auch Williams $(2000 ; 2010)$ heraus. 
geschränkt ist. Die gesamte Erfahrung von B geht in diesem Beispiel nicht über die Erfahrung mit den georderten Platten hinaus. Sie besteht darin, dass er darin geübt ist, sie auf den Ruf von A hin zu bringen. Seine Erfahrung in Bezug auf Platten, von der wir annehmen, dass sie seinen gesamten Erfahrungshorizont abdeckt, wird nicht durch mehr als zwei Züge in der Interaktion mit A bestimmt. Sie lehrt ihn in dieser Interaktion nicht mehr, als dass A ruft und er selbst daraufhin die Platte bringt. Darüber hinaus besitzt die Erfahrung nicht mehr an Bedeutung, die notwendig ist, um etwas aus ihr zu lernen.

Man könnte daraufhin einwenden, dass der Erfahrungsbegriff durch diese Sichtweise in ungerechtfertigter Manier reduziert würde. Tatsächlich müsste doch zugestanden werden, dass zu der Erfahrung des B in der Interaktion mit A noch viele weitere Aspekte hinzutreten als nur die beiden genannten. $\mathrm{Zu}$ nennen wären hier beispielsweise die Empfindungen von B hinsichtlich des Gewichtes und der Oberflächenbeschaffenheit der Platte. Wäre nicht zu konstatieren, dass auch die Empfindungen des B Teil seiner Erfahrung mit Platten sind, die dazu geeignet wären, B die Überzeugung zuzuschreiben, dass die von ihm gebrachten Platten ein bestimmtes Gewicht und eine bestimmte Oberflächenbeschaffenheit aufweisen? Folglich wären Empfindungen ein ausgezeichneter Kandidat, um gute Gründe für infrage kommende Handlungen zu liefern. Dieser Verdacht ließe sich noch zusätzlich dadurch erhärten, dass sich Empfindungen als unbezweifelbare und nichtpropositionale Bedingungen der Interaktion zwischen A und B vorstellen ließen. ${ }^{16}$

Wenn man allerdings zulässt, dass andere Aspekte der Erfahrung in der Betrachtung berücksichtigt werden als diejenigen, die in der Interaktion tatsächlich eine von den Akteuren geteilte praktische Funktion übernehmen, dann führte dies zu den gleichen Problemen, die bereits in Bezug auf die epistemologische Tragweite empirischer Beobachtung festgestellt worden sind. Die semantische Bedeutung von Erfahrung resultiert aus einer gemeinsam geteilten Praxis. Solange die Empfindungen von B in der Interaktion mit A nicht in praktischer Hinsicht relevant werden, leisten sie keinen semantischen Beitrag zum Begriff der Erfahrung. ${ }^{17}$ Folglich gelangen wir mit der Argumentation zu der Schlussfolgerung, dass eine notwendige Bedingung dafür, dass die Erfahrung etwas zu

16 David Hume scheint beispielsweise im Hinblick auf seine Theorie der unmittelbaren Affekte eine starke phänomenologische Auffassung zu vertreten, die mit einem unmittelbaren Bewusstsein der Empfindung von Wünschen verbunden ist; vgl. Hume (1978), 415; dazu auch Stroud (1977).

17 Vgl. in dieser Hinsicht auch die Bemerkungen in Bezug auf die Möglichkeit einer Privatsprache in Wittgenstein (2009), 243 ff. (im Folgenden zitiert als PU). 
lehren imstande ist, darin besteht, dass sie ein aktiver Bestandteil einer gemeinsam geteilten Praxis ist.

Ein zweiter Einwand könnte auf die Behauptung hinauslaufen, dass es sich im Hinblick auf die Interaktion zwischen A und B aufgrund der strengen Einschränkungen auf gerade einmal zwei Züge nicht um eine sprachliche Interaktion handeln kann. Primitive Sprachspiele im Sinne Wittgensteins geben keine geeigneten Beispiele für ein Handeln ab, das Gründen unterliegt, da das Geben und Annehmen von Gründen einer argumentativen Praxis zugehörig ist, die eine propositionale Struktur voraussetzt. In dieser Hinsicht hat Robert Brandom angemerkt, dass

the ,Slab' Sprachspiel that Wittgenstein introduces in the opening sections of the Philosophical Investigations should not, by the standards of demarcation, count as a genuine Sprachspiel [...]. It is a vocal but not yet a verbal practice. By contrast to Wittgenstein, the inferential identification of the conceptual claims that language (discursive practice) has a center; it is not a motley. ${ }^{18}$

Brandoms inferentialistische Sichtweise und die damit verbundene Eingrenzung des Sprachbegriffs steht in gewisser Weise quer zu Wittgensteins Konzeption von Sprache. Seine Verpflichtung auf die Unterscheidung zwischen „vocal“ und „verbal“ steht allerdings vor der äußerst schwierigen Situation, den Zusammenhang zwischen der Natur nicht-inferentiell agierender Wesen und dem Phänomen inferentialistisch strukturierter Rationalität zu erklären. ${ }^{19}$ Die Fokussierung auf den propositionalen Aspekt der Sprache als verbaler Praxis bedeutet nicht nur eine enorme Verkürzung des Sprachbegriffs, sondern lässt auch eine schier unüberbrückbare Kluft dort entstehen, wo die Entstehung von Rationalität zu erklären ist. Demgegenüber lässt sich Wittgensteins Idee primitiver Sprachspiele dienstbar machen, um Rationalität genalogisch als eine ständige Erweiterung der Sprachfähigkeit ausgehend von primitiven Sprachspielen zu betrachten. Auf diese Weise entsteht die Möglichkeit von Praktiken, die man unter dem Gesichtspunkt der Erweiterung als „elaborierte“ Sprachspiele bezeichnen kann. ${ }^{20}$

Offensichtlich besteht ein Unterschied zwischen nicht anzweifelbaren und nichtpropositionalen Gründen einerseits und den propositionalen Äußerungen von Gründen andererseits. Gute Gründe manifestieren sich in primitiven Sprachspielen, während propositionale Wissensansprüche und darauf bezogene

18 Brandom (2000), 14 (Hervorhebungen im Original).

19 Vgl. dazu auch Tolksdorf (2011).

20 Zu dieser Terminologie vgl. Kober (1993). Zur Idee der Erweiterung primitiver Sprachspiele vgl. Wittgenstein (1984a), 151 (im Folgenden zitiert als BPP) und ders. (1989), 115 (im Folgenden zitiert als UW); vgl. dazu auch Tolksdorf (2011) und Hacker (2007), 221 ff. 
Gründe als elaborierte Sprachspiele verstanden werden können. In einer Bemerkung vom 12.10.1937 macht Wittgenstein die Unterscheidung der beiden Arten und ihren Zusammenhang sehr deutlich: „Die Grundform unseres Spiels muss eine sein, in der es den Zweifel nicht gibt“ (UW 105, Hervorhebung im Original). Und einen Tag später schreibt er:

Wir sagen: „Nimm diesen Sessel!“ und es kommt uns nie in den Sinn, dass wir uns irren könnten, dass es vielleicht kein Sessel ist, dass spätere Erfahrung uns etwas anderes lehren könnte. Ein Spiel wird hier gespielt ohne die Möglichkeit des Irrtums, und ein anderes komplizierteres mit dieser Möglichkeit. (ebd.)

Eine weitere erhellende Bemerkung in dieser Hinsicht wird am 21.10.1937 notiert: „Der Ursprung und die primitive Form des Sprachspiels ist eine Reaktion; erst auf dieser können die komplizierteren Formen wachsen. [Absatz] Die Sprache - will ich sagen - ist eine Verfeinerung, ,im Anfang war die Tat““(UW 115). ${ }^{21}$

Diese Bemerkungen belegen, dass primitive Sprachspiele, wie Wittgenstein zu Beginn seiner Philosophischen Untersuchungen deutlich macht, nicht alles sind, was wir eine Sprache nennen, ${ }^{22}$ sondern dass eingeübte Sprachspiele den Ausgangspunkt bilden, von dem aus die Praxis weiterer, elaborierter Sprachspiele erlernt wird. Die Fähigkeit, Gründe propositional zu äußern, setzt von daher einiges mehr voraus, als man zunächst geneigt ist anzunehmen. Gute Gründe manifestieren sich in primitiven Praktiken, während das Geben und Annehmen propositionaler Gründe in Form elaborierter Praktiken vollzogen wird, die aus den primitiven Praktiken hervorgehen. Man folgt einer verkürzten Sichtweise, wenn man davon ausgeht, dass sich die Bedeutung von Gründen aus dem propositionalen und inferentiellen Prozess der Argumentation selbst heraus erschöpfe. Vielmehr resultiert die Fähigkeit, Gründe geben und annehmen zu können, aus einem primitiven Vorgang des Einübens und Erlernens einer geteilten Praxis, wie man unter bestimmten Umständen Gründe gibt und was man unter diesen Umständen als Gründe akzeptiert ${ }^{23}$.

21 Hinsichtlich des Faust-Zitats vgl. auch ÜG 402. Es ist eine äußerst interessante Beobachtung, dass Sigmund Freud seine Schrift Totem und Tabu mit eben diesem Zitat beschließt und es dabei in einem ganz ähnlichen Zusammenhang verwendet: „Der Primitive ist ungehemmt, der Gedanke setzt sich ohneweiters in Tat um, die Tat ist ihm sozusagen eher ein Ersatz des Gedankens, und darum meine ich, ohne selbst für die letzte Sicherheit der Entscheidung einzutreten, man darf in dem Falle, den wir diskutieren, wohl annehmen: Im Anfang war die Tat.“ Freud (2007), 216-217. 22 Vgl. PU 3.

23 ÜG 18: ,,Ich weiß es‘ heißt oft: Ich habe die richtigen Gründe für meine Aussage. Wenn also der Andre das Sprachspiel kennt, so würde er zugeben, dass ich das weiß. Der Andre muss sich, 
Die Sozialisierung in gemeinsame Praktiken erfolgt unter Anleitung eines Lehrers im Erlernen von Sprachspielen durch Erfahrung „unter normalen Umständen“ (ÜG 27). ${ }^{24}$ Primitive Sprachspiele, wie das Beispiel vom Bauenden und seinem Gehilfen, sind exemplarisch für Interaktionen, in denen Kinder ihre Muttersprache erlernen. „Solche primitive Formen der Sprache verwendet das Kind, wenn es sprechen lernt. Das Lehren der Sprache ist hier kein Erklären, sondern ein Abrichten“ ${ }^{25}$ notiert Wittgenstein. Dass Kinder in dieser Weise in den primitiven Sprachgebrauch eingeübt werden, führt dazu, dass infolge des eingeübten Sprachgebrauchs ihre Erfahrung zum ersten Mal Bedeutung erhält. „Es muss uns etwas als Grundlage gelehrt werden“ (ÜG 449), bemerkt Wittgenstein, und führt daraufhin fort: „Ich will sagen: Unser Lernen hat die Form ,Das ist ein Veilchen“, ,Das ist ein Tisch““ (ÜG 450).

Wir können aus dem Gesagten schließen, dass die Erfahrung eines Veilchens für ein Kind noch mit keinerlei semantischer Bedeutung verbunden ist, bevor es in darauf bezugnehmende geteilte Praktiken eingeführt worden ist. Erst im Rahmen geteilter Praktiken wird die Erfahrung eine semantisch greifbare. Dazu muss nicht notwendig vorausgesetzt werden, dass sie einen verbalen Ausdruck erfährt. Auch in unausgesprochener Form ist sie in der Lage Erfahrungssätze zu stützen, wie Wittgenstein bemerkt: „Wir lernen als Kinder Fakten, [...], und wir nehmen sie gläubig hin. Ich glaube, dass es eine Insel, Australien, gibt von der und der Gestalt usw. usw., [...]. Dieser Glaube mag nie ausgesprochen, ja, der Gedanke, dass es so ist, nie gedacht werden“ (ÜG 159).

Ich möchte noch einmal zusammenfassen, inwiefern die primitiven Einübungspraktiken eine Antwort auf die Frage geben, wie es zu verstehen ist, dass Akteure im Besitz nichtpropositionaler guter Gründe sind. Wie bereits beschrieben worden ist, sind innerhalb dessen, was Wittgenstein ein System von empirischen Sätzen nennt, Sätze vorhanden, die die Form empirischer Sätze aufweisen, wie beispielsweise „Das ist ein Veilchen“, die aber tatsächlich nicht als solche verwendet werden. Sie besitzen nicht die Eigenschaft, bezweifelt werden zu können, sondern vielmehr besteht ihre Funktion innerhalb des

wenn er das Sprachspiel kennt, vorstellen können, wie man so etwas wissen kann. “ Hervorhebung im Original.

24 Es ist wichtig zu berücksichtigen, dass die Praktiken durch Erfahrung erlernt werden und nicht durch das Befolgen expliziter Regeln. Zwar könnte man annehmen, dass explizite Regeln als geeignete Kandidaten für ein Verständnis guter Gründe in Frage kommen. Doch hier stößt man wiederum auf das Problem der Interpretation. Wie ist der Ausdruck einer expliziten Regel zu interpretieren? Auch in dieser Frage zeigt sich, dass eine Regel ihre Bedeutung aus einer gemeinsamen Praxis heraus gewinnt (vgl. ÜG 44; auch PU 198 ff.).

25 PU 5; vgl. auch PU 7. 
Systems darin, den zweifelsfreien Hintergrund $\mathrm{zu}$ bilden, vor dem grundsätzlich anzweifelbare Beobachtungssätze erst geäußert werden können. Auf diese Funktion sind Sätze nicht ontologisch festgelegt. Die Funktion hängt vielmehr davon ab, in welcher Weise Sätze in einem Sprachspiel verwendet werden. ${ }^{26}$ Es sei richtig, betont Wittgenstein, „dass der gleiche Satz einmal als von der Erfahrung zu prüfen, einmal als Regel der Prüfung behandelt werden kann“ (ÜG 98). Der Satz „Das Wasser siedet bei $100{ }^{\circ} \mathrm{C}$ “ kann zum einen als Erfahrungssatz dienen, mit dem ausgedrückt wird, dass in einer bestimmten Situation erhitztes Wasser beginnt zu sieden. Diese Gebrauchsweise setzt voraus, dass man die regelgeleitete Verwendung der Ausdrücke „Wasser“, „sieden“, „erhitzen“, „Temperatur“, etc. in praktischen Zusammenhängen erlernt hat. Ihre Bedeutung ergibt sich indes nicht durch die gemessene Temperatur. Das zeigt sich auch dadurch, dass der Satz zum anderen auch als Regel verwendet werden kann, mit der exemplifiziert wird, was wir beispielsweise unter der Bedeutung von „100 ${ }^{\circ} \mathrm{C}$ “ verstehen. Es steht demzufolge nicht von vornherein fest, wie der Satz zu verwenden ist, und damit bleibt zunächst offen, was wodurch zu prüfen ist (vgl. ÜG 125). Außerdem fügt Wittgenstein dieser Feststellung hinzu, dass es keine scharfe Grenze zwischen einer Regel und einem Erfahrungssatz gebe (ÜG 319). Wir können daraus folgern, dass Erfahrung in einem weiteren Sinn die Grundlage sowohl für Erfahrungssätze wie auch für Regeln darstellt, und die Funktion, die der geäußerte Satz im System einnimmt, von der Rolle abhängt, die er im Sprachspiel spielt.

Diese Überlegung führt zu einem interessanten Punkt in Bezug auf primitive Sprachspiele, mit denen ein Kind in die gemeinsame Praxis eingeführt wird. Wie das Beispiel des Bauenden und seines Gehilfen gezeigt hat, ist ein Kind, das nur wenige Sprachspiele spielt, in der Möglichkeit, seiner Erfahrung eine semantische Bedeutung zu geben, in hohem Maße eingeschränkt. Es befindet sich in genealogischer Hinsicht noch gar nicht an dem Punkt, die Ressourcen bereitzustellen, die bezweifelbare Erfahrungssätze stützen könnten. Um Sätze wie z. B. „Das ist ein Veilchen“ als Regeln zu verwenden, muss es vorerst in primitive Praktiken eingeübt werden. Unter diesen Bedingungen ist das Kind vorerst noch gar nicht in der Lage, die Praktiken, in die es eingeführt wird, zu bezweifeln. Es hat noch keinen bestehenden Hintergrund, von dem aus es die ihm antrainierten Praktiken als richtig oder falsch bzw. die sie beinhaltenden Äußerungen als wahr oder falsch beurteilen könnte. Im Hinblick auf primitive Sprachspiele optiert das Kind nicht für eine Vorgehensweise, sondern es reagiert lediglich auf die Erfahrung,

26 Entgegen dieser Feststellung argumentiert Moyal-Sharrock (2007) für das Bestehen von „universal hinges“. Vgl. dazu meine Kritik an dieser Annahme in Franken (2011). 
die es im Zusammenhang mit dem Einüben macht. Da die Möglichkeit eines überlegten Urteils für das Kind nicht besteht, muss es dem Lehrenden in der Weise des Einübens schlichtweg vertrauen. ${ }^{27}$ Das Einüben ist demzufolge dadurch ausgezeichnet, dass es außerhalb jeglicher Kritik steht und den Praktiken gerade dadurch eine Sicherheit abseits irgendeines Zweifels zuteilwird.

Daraufhin kann nun abschließend die Folgerung gezogen werden, dass durch das Vertrauen des Kindes und seinen damit verbundenen zweifellosen und blinden Gehorsam, von dem das Einüben in primitive Sprachspiele begleitet wird, der weitere Spracherwerb auf der Grundlage unzweifelhafter und infolgedessen guter Gründe ermöglicht wird. Wittgenstein formuliert diesen Punkt wie folgt: „,[D]as Ende [der Begründungen] ist nicht die unbegründete Voraussetzung, sondern die unbegründete Handlungsweise“ (ÜG 110).

\section{2}

Durch das Vorangegangene ist deutlich geworden, wie und warum es möglich ist, dass Menschen die Sätze der Physik nicht als ein Wissen betrachten können, aus dem Handlungsgründe hervorgehen, und das für sie demzufolge faktisch in vielen Zusammenhängen auch nicht als ein handlungsleitendes Wissen zählt. Es ist vor diesem Hintergrund überhaupt nichts Geheimnisvolles an der Tatsache, dass Angehörige einer anderen Kultur statt den Sätzen der Physik den Sätzen eines Orakels vertrauen. Damit zeigen sie, dass sie auf eine andere Weise gelernt haben zu urteilen. Die Weise, in der sie es gelernt haben, ist gestützt durch Sätze, die unterschiedlich sind zu denen, die Mitglieder aus anderen Kulturen gelernt haben. Dennoch besteht kein Zweifel daran, dass sie in der Lage sind, den Akteuren im Hinblick auf ihre Handlungen gute Gründe zu liefern.

Vor diesem Hintergrund ist Wittgensteins eingangs erwähntes Gedankenexperiment der beiden Gruppen verbunden mit der Frage, wie ihre verschiedenen Praktiken einerseits von dem Standpunkt derer beurteilt werden können, die den Sätzen der Physik vertrauen (ich werde sie im Folgenden die Gruppe A nennen), und andererseits von denen, die dem Orakel vertrauen (Gruppe B). Nachdem er in ÜG 608 die rhetorische Frage geäußert hat, ob es denn falsch sein könne, sich in seinem Handeln nach den Sätzen der Physik zu richten, fragt er nun, ob es aus der Sicht der Gruppe A falsch sein könne, sein Handeln nach den Sprüchen von Orakeln auszurichten (ÜG 609). Nach der bisherigen Betrach-

27 Instruktiv in diesem Zusammenhang ist nach wie vor Hertzberg (1988). 
tung wird deutlich, dass in beiden Fällen die Sinnhaftigkeit der Frage, ob die geteilte Praxis als wahr oder falsch beurteilt werden kann, von Grund auf auf wackeligen Füßen steht: Denn aus den vorangegangenen Überlegungen haben wir erfahren, dass es gerade im Wesen guter Gründe liegt, dass sie vom Zweifel ausgeschlossen sind, und mithin folglich auch davon, Gegenstand einer Beurteilung zu sein.

Wir werden nun den Überlegungen des Gedankenexperiments in ihrer mutmaßlichen Intention folgen, indem wir die Rolle von propositional strukturierten Gründen untersuchen. Dabei werden wir die eben gemachte Schlussfolgerung hinsichtlich der Beurteilung der von beiden Gruppen verfolgten Praktiken für einen Moment zurückstellen und dem als Irrtum herausgestellten Gedankengang folgen. Denn Wittgenstein fragt probehalber, wie wir es uns vorzustellen haben, dass Mitglieder der Gruppe A - ungeachtet der Tatsache, dass gute Gründe einer Beurteilung unzugänglich sind - die Praktiken der Gruppe B als falsch und fehlgeleitet beurteilen. Die Mitglieder der Gruppe A, so die Vorstellung, sind Opponenten der Mitlieder von Gruppe B. Unter diesen Bedingungen ist der erste bemerkenswerte Aspekt im Hinblick auf die rhetorische Frage am Ende von ÜG 609, dass Mitglieder der Gruppe A ihr Sprachspiel als ein Fundament betrachten, von dem aus sie versuchen, ein Verständnis der fremden Praktiken zu erreichen, und auf dem sie die Beurteilung vornehmen. Aus dieser Feststellung heraus lassen sich drei weitere Frage ableiten, die es zu klären gilt: (i) Welches Sprachspiel ist hier gemeint? (ii) Warum lässt sich das Sprachspiel als „das ihrige“ bezeichnen? (iii) In welcher Hinsicht lässt sich das Sprachspiel als ein Fundament verstehen? Ich werde diesen drei Fragen im Folgenden nachgehen.

Im vorangegangenen Abschnitt ist dafür argumentiert worden, dass die Teilnehmer einer Sprachgemeinschaft ihre ersten Sprachspiele in Übungsinteraktionen erlernen, die Wittgenstein ,primitiv“ nennt. Ein Lehrer äußert beispielsweise den Satz „Das ist ein Veilchen“ und das Kind reagiert auf diese Äußerung, indem es sie wiederholt. Dieses Sprachspiel kann ein Ausgangspunkt für viele weitere sprachliche Züge sein, die Teil einer gemeinsamen Praxis in einer Sprachgemeinschaft werden. Im Hinblick auf das Sprachspiel lässt sich zunächst einmal feststellen, dass dem Kind, neben einer Reihe anderer Dinge, beigebracht wird, wie zu urteilen ist. In ähnlicher Weise lernen die Mitglieder der beiden Gruppen A und B die Praxis des Urteilens auf eine primitive Weise, wenn sie in die Sprachgemeinschaft eingeführt werden. Folglich ist in der Beantwortung der ersten der drei Fragen die Annahme plausibel, dass (i) das Sprachspiel, auf das sich Wittgenstein in ÜG 609 bezieht, jenes ist, das wir „urteilen“ nennen.

Der entscheidende Punkt im Hinblick auf das Gedankenexperiment liegt jedoch darin, dass die Mitglieder beider Gruppen in unterschiedlicher Weise gelernt haben zu urteilen. Die Mitglieder der Gruppe A haben inter alia die Sätze 
der Physik gelernt, die sie dazu verwenden, bestimmte Erfahrungsurteile abzugeben. Demgegenüber haben Mitglieder der Gruppe B gelernt, dass Orakel existieren, an deren Aussagen sie ihr Handeln orientieren und von denen sie ihre Urteile abhängig machen. Beide Gruppen haben, wie sich gezeigt hat, nichtpropositionale gute Gründe, die sie auf propositionalem Wege dadurch beschreiben könnten, dass sie sagten: „Ich habe von Kind auf so urteilen gelernt. Das ist Urteilen [ÜG 128]. So habe ich urteilen gelernt; das als Urteil kennengelernt [ÜG 129].“28

Vor diesem Hintergrund wird ebenfalls deutlich, in welcher Hinsicht man (ii) davon sprechen kann, dass das Sprachspiel der beiden Gruppen A und B „das ihrige“ sei. Denn es ist jeweils die in ihrer Sprachgemeinschaft eigentümlich geregelte Weise, in der sie eingeübt haben, gewisse primitive Praktiken zu verwenden, die sie „urteilen“ nennen. In einer gewissen Hinsicht kann man daher (iii) sagen, dass primitive Praktiken das Fundament für weitere Sprachspiele bilden. ${ }^{29}$ Auf diese Weise haben die Mitglieder beider Gruppen gelernt zu urteilen. Es ist ihre unbegründete Weise zu handeln. Und in dieser Hinsicht können die primitiven Praktiken als Ausgangspunkt angesehen werden, von dem aus die Akteure der beiden Gruppen jeweils in der geregelten Weise ihre Urteilsspiele spielen, so, wie sie in ihrer jeweiligen Sprachgemeinschaft praktiziert werden.

Diese Überlegung führt zu dem zweiten bemerkenswerten Punkt an Wittgensteins rhetorischer Frage: Die primitive Praxis als Fundament wird als Ausgangspunkt des Versuchs betrachtet, den Gegner in Bezug auf seine Urteilspraxis zu bekämpfen. Wie einleitend unterstellt worden ist, steht die Frage, wie der Konflikt zwischen den beiden Gruppen verstanden werden kann, im Zentrum des Abschnitts ÜG 608-612. Wenn man nun unter diesem Aspekt versucht, ein Verständnis des Ausdrucks ,bekämpfen‘ zu erreichen, dann lassen sich kurzerhand die folgenden Beobachtungen hinsichtlich der Ursache und des Zwecks seiner Verwendung anstellen: Erstens wird der im Kampf ausgetragene Konflikt durch eine Verschiedenheit in den primitiven Praktiken hervorgerufen, wie wir bereits gesehen haben. Zweitens scheint der Zweck bzw. das Ziel des Bekämpfens in der Beurteilung der Praktiken der jeweils anderen Gruppe zu liegen, mit dem Ergebnis, dass sie, aufgrund ihrer Verschiedenheit, als falsch und unkorrekt beurteilt

28 Hervorhebungen im Original. Obwohl sich gute Gründe propositional beschreiben lassen, bleibt zu beachten, dass sie an sich von nichtpropositionaler Natur sind. Vielmehr ist es die Beschreibung guter Gründe, die eine propositionale Struktur besitzt.

29 In Franken (2011) habe ich dafür argumentiert, dass die diesbezügliche Rede von „Fundamenten“ zu bestimmten Missverständnissen einer Interpretationsrichtung führt, die als „foundationalism“ bekannt geworden ist. Darauf kann hier nicht weiter eingegangen werden. Der entscheidende Punkt an dieser Stelle ist, dass primitive Sprachspiele nicht als unwandelbare und universal geltende Fundamente verstanden werden sollten. 
werden. Das Bekämpfen richtet sich daher gegen eine bestimmte Praxis, und demzufolge scheint es klar zu sein, dass sein Zweck überhaupt nur dann sinnvoll verstanden werden kann, wenn es die Möglichkeit zulässt, die Differenzen einzuebnen, die zu dem Konflikt geführt haben. Wir müssen uns demzufolge die Frage stellen, ob die Ursachen des Konfliktes tatsächlich zulassen, dass sein Zweck erreicht werden kann. Ist es tatsächlich vorstellbar, dass unter den Gruppen mit sprachlichen Mitteln ein Sieg errungen werden kann?

Um diese Frage zu beantworten, kehren wir zurück zu der Feststellung, die wir zwischenzeitlich aus argumentationstechnischen Gründen heraus beiseite gestellt haben, nämlich, dass beide Gruppen aus ihrer Praxis heraus bereits gute Gründe besitzen, die über den Zweifel und die Beurteilung erhaben sind. Vor diesem Hintergrund spielen beide Gruppen verschiedene Sprachspiele und es ist unter diesen Bedingungen nicht einzusehen, wie das Ziel des zur Debatte stehenden Kampfes jemals erreicht werden könnte. Bei genauem Blick stellt man nämlich fest, dass das Austragen des Konfliktes mit sprachlichen Mitteln und mit dem Ziel der Übernahme eigener Praktiken durch die attackierte Partei in Gänze seinen Sinn verfehlt, weil unter den gegebenen Bedingungen das Ziel aus logischen Gründen nicht erreicht werden kann.

In diesem Zusammenhang besteht die Subtilität der Frage in ÜG 610 nicht in der Klärung des Sachverhalts, ob es recht oder unrecht ist, den jeweils anderen zu bekämpfen, sondern vielmehr in der Folgerung, dass bereits die Frage an sich ihren Sinn verfehlt. Weder macht es Sinn zu fragen, ob es richtig oder falsch ist, einer primitiven Praxis zu folgen, noch, ob es richtig oder falsch ist, sie zu bekämpfen. Die primitive Praxis, verstanden als Ausgangspunkt, von dem aus der andere bekämpft wird, stellt keine effektiven Mittel zur Verfügung, um das Ziel des Kampfes zu erreichen. „Freilich“, so bemerkt Wittgenstein vorausschauend mit einem ironischen Unterton, wird man das „Vorgehen mit allerlei Schlagworten (slogans) aufstützen“. Die ernüchternde Wahrheit jedoch ist, dass solche „slogans“ nichts anderes sind als hölzerne Eisen.

An einer anderen Stelle seiner Notizen in Über Gewissheit führt Wittgenstein aus, dass man sich eine Person vorstellen könne, die in einer bestimmten Hinsicht „mit [...] meiner Überzeugungen [...] im Widerspruch ist. Und da könnte es sein, dass [sie] meinen Grundanschauungen widerspricht. Und wäre es so, so müsste ich's dabei bewenden lassen“ (ÜG 238, Hervorhebung im Original). Stimmt man dieser Folgerung zu, so scheint es im Allgemeinen ein unglücklicher Zug des Menschen zu sein, dass er in der Mehrzahl der Fälle weder die Überflüssigkeit an seinem Versuch erkennt, eine andere Sichtweise mit sprachlichen Mitteln zu bekämpfen, noch dass er sich nicht dem Drang entgegenstellen kann und sich dazu entschließt, es bei der Situation bewenden zu lassen. Wittgensteins Kritik in ÜG 611 zielt in direkter Weise auf diesen Punkt ab. 
Zur Kenntnis nehmend, dass aus dem Konflikt für beide Parteien kein befriedigender Weg herauszuführen scheint, und sich damit das Dilemma zwischen der Tatsache, dass es Unterschiede gibt in der Weise, wie primitive Sprachspiele eingeübt werden, und der Forderung, diese Unterschiede einzuebnen, fortsetzt, wird in ÜG 612 eine weitere Variante in Betracht gezogen: „[W]ürde ich ihm denn nicht Gründe geben?“30 Die Praxis des Gebens und Annehmens von Gründen scheint zunächst ein vielversprechendes Mittel zu sein, um den Versuch fortzusetzen, die Verschiedenheiten durch die Kraft von Argumenten einzuebnen. Interessanterweise geht die Praxis des Gebens und Annehmens von Gründen auf

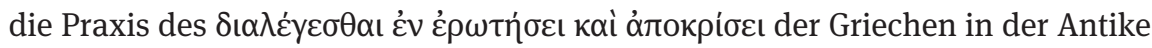
zurück, die als eine Form des Frage-Antwort-Spiels im Rahmen eines Wettbewerbs zwischen einer fragenden Person A und einer antwortenden Person B zu verstehen ist. ${ }^{31}$ Dieser Vorgang wird detailliert im achten Buch der Aristotelischen Topik geschildert. Das Ziel des Spiels bestand darin, den Mitspieler B in seiner Antwort entweder auf einen Widerspruch im Hinblick auf seine These oder entsprechende Nebenannahmen zu verpflichten, oder aber, ihn dahin zu bringen, etwas abzustreiten, das aus seiner These folgte. War die Antwort des Mitspielers in einer dieser Hinsichten problematisch, dann wurde A damit zum Sieger erklärt.

Behalten wir den Blick für einen Moment noch auf diesem historischen Beispiel für das Geben und Annehmen von Gründen und fragen nach den Bedingungen, unter denen das Spiel ausgetragen wird. Hier ist zunächst festzustellen, dass das Spiel bestimmte Regeln besitzt, nach denen gespielt wird, und die entscheiden, wer zum Sieger erklärt wird. Eine dieser Regeln lautet „B verliert das Spiel, wenn er sich zu seinen Thesen in einen Widerspruch begibt“. Betrachtet man genauer, wie auf der Grundlage dieser Regel das Spiel entschieden werden kann, dann wird deutlich, dass, um den Widerspruch feststellen zu können, auf weitere Regeln, die Anwendung in einer bereits bestehenden Praxis finden, zurückgegriffen werden muss.

Wie ist das zu verstehen? Ein Widerspruch muss in der Beziehung zweier Aussagen festgestellt werden, die sich gegenseitig widersprechen. So sagt der Satz vom Widerspruch, dass zwei einander widersprechende Aussagen nicht zugleich zutreffen können. ${ }^{32}$ Den sich widersprechenden Aussagen liegt, wie oben festgestellt wurde, ein regelgeleitetes Verhalten zugrunde, das mit den Sprachteilnehmern eingeübt worden ist. Nähmen wir an, dass sich bereits Äußerungen in der Praxis primitiver Sprachspiele widersprächen, dann wäre das resultierende Verhalten in hohem Maße impraktikabel und unverständlich. Wie sollte es zu ver-

30 Hervorhebung im Original.

31 Vgl. dazu Mann (1992).

32 Vgl. Aristoteles (1995), 1005b. 
stehen sein, dass ein Kind zugleich die Überzeugungen erwirbt, dass etwas ein Veilchen und kein Veilchen ist? Wie soll sich das Kind mit diesen sich widersprechenden Überzeugungen verständlich machen? Das System, von dem Wittgenstein spricht, muss demzufolge einen gewissen Grad an Konsistenz aufweisen. ${ }^{33}$ Ist dieser Grad nicht erfüllt, dann beginnt die Person sich und anderen in ihrem Verhalten unverständlich zu werden. Dies ist ein Hinweis darauf, dass das Verhalten der Person zur Verständigung unbrauchbar geworden ist und es damit seinen Selbstzweck, nämlich den der Verständigung, zumindest partiell verliert. Man kann auch davon sprechen, dass es das notwendige Kriterium der Praktikabilität, d. h. seinen praktischen Zweck der Verständigung, nicht länger erfüllt. ${ }^{34}$ Die entscheidende Bedingung zur Niederlage im Spiel der Griechen ist demzufolge die Nichterfüllung einer für das Alltagsleben des Menschen essentiellen Bedingung, nämlich Praktikabilität. Wenn diese fehlt, ist das Spiel zwangsläufig beendet, da keine praktische Möglichkeit zur Fortsetzung des Spiels mehr besteht. Das Spiel ist aus, der Gegner hat sich in den Möglichkeiten seines Verständlichmachens selbst matt gesetzt.

Wenn man die historischen Wurzeln des Spiels vom Geben und Annehmen von Gründen in Betracht zieht, dann offenbart sich der spielerische Charakter der Interaktionspraxis. Hinzu tritt das erklärte Ziel des Spiels, den Gegner in einer Art kämpferischer Auseinandersetzung zu besiegen. Wie wir gesehen haben, muss bei dieser Betrachtung jedoch das Missverständnis umgangen werden, dass der nach Gründen fragende Teilnehmer seinen Mitspieler in letzter Instanz aktiv besiegt, denn aktive Mittel stehen ihm dazu nicht zur Verfügung. Vielmehr ist es der Antwortende, der sich in Widersprüche verstrickt und sich dadurch letztlich selbst besiegt.

Infolge der Bedingungen, die uns das griechische Modell verdeutlicht hat, ist es interessant zu überlegen, was aus ihnen für das Verständnis des Kampfes in Wittgensteins Gedankenexperiment folgt. Zunächst müssen wir die uns angebotene Perspektive dazu umkehren. Der Vorgang des Überzeugens ist nach dem griechischen Vorbild nicht in der Weise zu konstruieren, dass die Gruppe A, die die Sätze der Physik in primitiven Praktiken erlernt hat, nun versucht, Gründe für den Vorgang ihrer Praxis anzugeben, um die Abkehr der Gruppe B von ihren

33 ÜG 102: „Könnte ich nicht glauben, dass ich einmal, ohne es zu wissen, etwa im bewusstlosen Zustand, weit von der Erde entfernt war, ja, dass Andre dies wissen, es mir aber nicht sagen? Aber dies würde gar nicht zu meinen übrigen Überzeugungen passen. Nicht, als ob ich das System dieser Überzeugungen beschreiben könnte. Aber meine Überzeugungen bilden ein System, ein Gebäude.“

34 Vgl. Franken (2013; 2014). 
Praktiken zu forcieren. Vielmehr wird sie von der Gruppe B Gründe einfordern, die die Durchführungen ihrer Praktiken rechtfertigen. Die Versuche der Gruppe A, die Praktiken der Gruppe B durch überzeugende Gründe zu unterbinden, setzt indes voraus, dass sie die Sprachspiele spielen können, die sich aus den primitiven Praktiken heraus entwickelt haben. Wie wir jedoch gesehen haben, ist ihr Umgang mit den Sätzen der Physik ein vollkommen anderer, als es bei den Kontrahenten der Fall ist. Infolgedessen ist es schwer einzusehen, wie eine solche Auseinandersetzung ihren Zweck erfüllen könnte. Die primitiven Praktiken der Gruppe A, die sich zu den ,ihrigen“ Sprachspielen des Gebens und Annehmens von Gründen erweitert haben, können nicht ohne weiteres von der Gruppe B verstanden und folglich nicht akzeptiert werden.

Gleiches gilt nach dem griechischen Modell umgekehrt für die Akzeptanz der Rechtfertigungen von Gruppe B durch die Gruppe A. Das Modell zeigt, dass die Rechtfertigungen, die von der Gruppe B gegeben werden, nicht vor der Beurteilung der Gruppe A zu bestehen haben, sondern in einem kohärenten Verständnis mit den eigenen Praktiken stehen müssen, um durchgängig verständlich zu sein. Auf das Verständnis der Rechtfertigungen von Seiten der Gruppe A besitzt die Gruppe B letzten Endes ebenso wenig Einfluss. Vor diesem Hintergrund reagiert Wittgenstein auf den in ÜG 612 gemachten Vorschlag, Gründe als überzeugendes Mittel heranzuziehen, mit der skeptischen Nachfrage: „[...] aber wie weit reichen die?“ Wir können nun die Antwort auf diese Frage unter Berücksichtigung der gegebenen Umstände ergänzen: Nicht hinreichend weit.

\section{3}

Wenn wir uns die Situation der beiden gegnerischen Parteien im Hinblick auf das Aufeinandertreffen ihrer den Konflikt provozierenden Praktiken betrachten, dann lässt sich das folgende Ergebnis feststellen: Das Bekämpfen kann, soweit wir gesehen haben, seinen Zweck nicht erfüllen, die gegnerische Partei dazu zu bewegen, sich von ihren Praktiken abzuwenden. Die Praxis des Gebens und Annehmens von Gründen muss in dieser Konfliktsituation notwendig scheitern.

So unbefriedigend das Resultat auch sein mag: Die gegnerischen Parteien sehen sich nach wie vor dem Dilemma gegenübergestellt, sich einerseits nicht mit den Unterschieden in der praktischen Umgangsweise zufriedengeben zu können, und andererseits nicht in der Lage zu sein, diese Unterschiede auf dem Wege argumentativen Überzeugens einzuebnen. In Bezug auf die sich gegenüberstehenden Praktiken können propositionale Gründe schlichtweg keine Entschei- 
dung herbeiführen, da sie erst auf der Grundlage bereits geteilter Praktiken innerhalb einer Sprachgemeinschaft wirksam werden. Es zeigt sich demzufolge, dass es letzten Endes nur eine Antwort auf das Dilemma der beiden Parteien gibt, wenn sie es nicht einfach darauf beruhen lassen können, ihre Differenzen zur Kenntnis zu nehmen. Dieser ultimative Ausweg liegt meines Erachtens in der Überredung. Tatsächlich ist sie aber nicht nur als ein möglicher Ausweg anzusehen, sondern sie stellt sich tatsächlich bei näherem Hinsehen als der epistemologische Normalfall heraus, wenn wir uns selbst, der Welt und anderen gegenübertreten. Ich werde das im Folgenden erläutern.

Wie wir gesehen haben, beginnt der Prozess des Spracherwerbs mit primitiven Praktiken, in die ein Kind eingeübt wird. Zu dem Zeitpunkt, da das Kind in den Sprachgebrauch eingeübt wird, besitzt es noch keinen sprachlichen Hintergrund, vor dem es entscheiden könnte, ob es den richtigen oder inkorrekten, den wahren oder falschen Praktiken folgt. In primitiven Sprachspielen optiert das Kind nicht für eine bestimmte Verhaltensweise, es reagiert lediglich. Da sich vor diesem Hintergrund keine Möglichkeit einer überlegten Wahl ergibt, stellt sich das bloße Reagieren als wesentliches Element primitiver Praktiken heraus. Sie stützen die später hinzutretenden elaborierten Praktiken in epistemologischer Hinsicht und bleiben daher im Rahmen eines Interdependenzverhältnisses zu ihnen bestehen. ${ }^{35}$

Betrachtet man dieses Verhältnis genauer, dann zeigt sich, dass es auf zwei verschiedene Weisen gestört werden und das System damit partiell aus seinem epistemologischen Gleichgewicht gebracht werden kann: (i) Entweder treten neue Überzeugungen durch primitive Praktiken hinzu, die in einem praktischen Widerspruch zu bereits vorhandenen Überzeugungen stehen; oder (ii) ein Zweifel äußert sich im Rahmen elaborierter Sprachspiele, der die eigenen, unhinterfragt angenommenen Praktiken thematisch in Frage stellt. Auf keinem der beiden Wege kann die Störung durch die Äußerung eines Grundes erfolgen, der nicht aus den eigenen erlernten Praktiken hat hervorgehen können. Ein solcher Grund würde von der betreffenden Person schlichtweg nicht verstanden werden. Entweder die Person wird dazu herausgefordert, eine Änderung hinsichtlich der eigenen Überzeugungen vorzunehmen, die sie selbst einmal auf dem Wege primitiven Einübens erlernt hat, oder sie wird dazu herausgefordert, ihre auf primitivem Wege erworbenen Überzeugungen über den elaborierten Weg der sie erweiternden Sprachspiele selbst zu ändern. Diese erfüllen indes die Bedingung, dass sie verstanden werden, weil ihre Gebrauchsweise eigens erlernt worden ist.

35 Wittgenstein hat dieses Verhältnis durch verschiedene Metaphern zu beschreiben versucht; vgl. ÜG 96, 97, 152, 242. Ich werde auf sie hier aus Platzgründen nicht im Einzelnen eingehen. 
Da auf beiden Wegen letztlich eine Manipulation an den auf primitivem Wege erlernten Praktiken erfolgt, und primitive Praktiken gleichzeitig die Bedingungen für die Möglichkeit der Manipulation stellen, folgt daraus, dass auch ein Überzeugen durch Gründe letztlich eine Manipulation der auf primitivem Wege erworbenen Überzeugungen darstellt. Dieser Vorgang der Manipulation macht indes aus systematischer Sicht, wenn ich richtig sehe, den Kern der Bedeutung dessen aus, was wir unter dem Vorgang der Überredung verstehen. Überzeugen und Überreden stehen folglich in einem engen logischen Zusammenhang zueinander, der durch den Begriff der Manipulation primitiver Praktiken hergestellt wird.

Um den logischen Zusammenhang argumentativ zu unterfüttern, werde ich die beiden genannten Vorgänge einer Manipulation durch den Versuch des Überzeugens mit der eingangs erläuterten traditionellen Vorstellung einer Überredung vergleichen. Wir erinnern uns, dass der Vorgang des Überredens traditionell als eine Veränderung theoretisch oder praktisch relevanter Überzeugungen verstanden worden ist, die nicht unter das Ergebnis eines argumentativen Diskurses fällt. Der Vorgang des Überredens kann begrifflich demzufolge dadurch spezifiziert werden, dass er ohne die notwendige Akzeptanz einer argumentativen Rechtfertigung vollzogen wird. Um zu prüfen, inwieweit das Kriterium fehlender Akzeptanz kompatibel mit dem hier vorgestellten Begriff der Manipulation von Überzeugungen ist, werde ich es mit den beiden oben geschilderten Weisen der Manipulation konfrontieren.

(i) Die Situation, in der ein Mensch noch keine elaborierten Praktiken erlernt hat und sich noch in dem Stadium der Einübung primitiver Praktiken befindet, ist paradigmatisch für das lernende Kleinkind. Wenn die Einübung eines Kindes als Prozess des Überzeugens verstanden werden sollte, dann müsste die Akzeptanz des Erlernten ebenfalls eine Grundbedingung des Vorgangs sein. Wie wir jedoch gesehen haben, kann diese Bedingung im Rahmen der Einübung nicht erfüllt werden, da die Möglichkeit der Akzeptanz die Möglichkeit der Zurückweisung impliziert und darüber hinaus Kriterien bestehen müssen, unter denen Akzeptanz oder Zurückweisung gerechtfertigt werden kann. Diese Kriterien werden durch den sprachlichen Hintergrund geliefert, den das Kind allerdings erst durch das Einüben gewinnt. Akzeptanz und Zurückweisung gehören demgegenüber zu den elaborierten Praktiken, die einen solchen Hintergrund voraussetzen.

(ii) Die Situation ändert sich, wenn die Person über das Anfangsstadium des Spracherwerbs hinaus elaborierte Praktiken erlernen konnte und damit auch in die Lage versetzt worden ist, Gründe zu geben und entgegenzunehmen. Um in dieser Situation den Vorgang der Überredung deutlich zu machen, wollen wir exemplarisch annehmen, dass die betreffende Person sich dem Atheismus angeschlossen hat und nun von einem gläubigen Christen dazu überredet werden soll, 
mit ihm den Gottesdienst am kommenden Sonntag zu feiern. Der Atheist wird diese Einladung zunächst versuchen, freundlich abzulehnen, und beide werden gegenseitig Gründe dafür präsentieren, die dafür oder dagegen sprechen, den Gottesdienst aufzusuchen. Der Christ wird mit Texten aus der Bibel argumentieren, während der Atheist darauf insistieren wird, dass er nicht an Gott glaube und die Bibeltexte für ihn deshalb keine hinreichenden Handlungsgründe lieferten. Nehmen wir an, nach einiger Zeit käme der Atheist selbst darüber ins Zweifeln, ob er die Einladung so vehement hätte ausschlagen sollen. Obwohl die Texte des Neuen Testamentes keine Handlungsgründe für ihn liefern, entscheidet er sich dennoch, den Christen zu begleiten. Er wird dafür Gründe geltend machen, die er aus seiner eigenen Praxis heraus rechtfertigen kann. Unter diesen Bedingungen hat eine Manipulation seiner Handlungsentscheidung stattgefunden, ohne dass er die Argumente des Christen akzeptiert hat.

Aus diesen beiden Situationen heraus lässt sich schlussfolgern, dass die Veränderung des Systems von Überzeugungen entweder durch das Einüben von noch unbekannten Praktiken erfolgt, oder durch die Herausforderung bereits vorhandener Überzeugungen durch elaborierte Sprachspiele. In Wittgensteins Gedankenexperiment sind die Mitglieder der Gruppe B weder gewillt, neue Praktiken hinzuzulernen, noch ihre bisherige Praxis aufgrund von dargelegten Gründen zu ändern. Auch hier liegt eine Situation vor, in der jemand zur Änderung seiner Überzeugungen gebracht werden soll, ohne dass seine Akzeptanz eine Vorbedingung darstellt. Folgerichtig bemerkt Wittgenstein am Ende von ÜG 612: „Am Ende der Gründe steht die Überredung. “36

Im Hinblick auf die Gründe, aus denen eine Akteurin zu ihrer Handlung motiviert ist, hat John McDowell in dieser Richtung dafür argumentiert, dass die Akteurin durch Motive zu ihrem Handeln bewegt werden könne, die nicht bereits Bestandteil der motivationalen Verfassung der Akteurin sein müssen. ${ }^{37}$ Er meint, dass eine Person deshalb die richtigen Gründe für ihr Handeln in ethisch relevanten Situationen besitzt, weil sie die richtige Betrachtungsweise auf solche Situationen durch Erziehungsprozesse erwirbt, d. h. in geeignete Verhaltensweisen eingeübt wird. Eine Person lernt auf diese Weise, die Situation richtig zu beurteilen. Bei Personen, die nicht richtig erzogen worden sind, meint McDowell, dass die einzige Möglichkeit, solche Personen zu einer richtigen Sichtweise zu führen, darin besteht, sie zu bekehren:

36 Hervorhebung im Original.

37 Vgl. McDowell (1995). Er argumentiert damit gegen die Position des normativ existierenden Internalismus in Bezug auf Handlungsgründe, die von Autoren wie Bernard Williams vertreten wird. 
The idea of conversion would function here as the idea of an intelligible shift in motivational orientation that is exactly not effected by inducing a person to discover, by practical reasoning controlled by existing motivations, some internal reasons that he did not previously realize he had. ${ }^{38}$

Die einzige Möglichkeit, wie eine Person von der Richtigkeit einer Handlung überzeugt werden kann, ohne dass eine vorausgehende Motivation zu der Handlung vorausgesetzt wird, stellt laut McDowell der Prozess der Bekehrung dar. Die Person wird diesbezüglich, gewissermaßen nachträglich, in die Praktiken eingeübt, die notwendig sind, um die Überzeugungen und Motive zu erwerben, die einer (aus der Sicht McDowells) richtigen Betrachtungsweise entsprechen und folglich zu einem richtigen Handeln führen.

Auf dem gleichen Weg, wie der Lehrende das Kind in ein Sprachspiel einübt, das es noch nicht spielt, ist ein Mitglied der Gruppe A bemüht, sein Gegenüber dazu zu bringen, das Sprachspiel zu spielen, in das er selbst eingeübt worden ist. Konkret wird er versuchen, die Mitglieder der Gruppe B Sätze der Physik zu lehren. Der Unterschied zwischen den Mitgliedern der Gruppe B und Kleinkindern besteht indes darin, dass erstere bereits im Besitz von Praktiken sind, in die sie eingeübt worden sind, und die durch elaborierte Sprachspiele erweiterbar sind. Die Folge ist, dass der Versuch der Konfrontation mit neuen Praktiken dazu führen kann, dass die Gruppe B sich mit Mitteln elaborierter Praktiken zu verteidigen versucht. Fraglich ist, wie lange sie dem Druck der Überredung standhalten wird, denn das Zur-Wehr-Setzen erfordert eine konstante Aufmerksamkeit und damit einen hohen kognitiven Aufwand, auch wenn er letztlich nur in der Entscheidung zu bestehen scheint, sich in der eigenen Verhaltensweise stur zu stellen, um den Konflikt ohne eigene Veränderung zu überstehen. Letztlich, so möchte ich abschließend behaupten, werden wir im Allgemeinen, wie auch die Mitglieder der Gruppe B in der speziellen Situation, in der überwiegenden Mehrzahl der Fälle den druckvollen Versuchen zur Veränderung unserer Praktiken nachgeben, und zwar aus folgenden Gründen:

Erstens sind wir jeden Tag mit einer Mannigfaltigkeit eigener und fremder Praktiken konfrontiert. Viele von ihnen sind uns notwendigerweise unbekannt und erscheinen uns neu. Es ist leicht einzusehen, dass wir nicht mit jeder einzelnen Gegebenheit vertraut sind, die uns widerfährt. In dieser Hinsicht besteht die Notwendigkeit im Alltagsleben, sich durchgängig Übungssituationen auszusetzen. Zweitens finden viele dieser Einübungsvorgänge statt, ohne dass dies uns bewusst wäre. Oftmals ist es uns nicht bewusst, dass wir Praktiken mit guten Gründen ausführen, die uns beispielsweise durch die spitzfindige und multime-

38 Ebd., 74, Hervorhebung im Original. 
dial agierende Werbeindustrie zur Verfügung gestellt werden. Der Erfolg kommerzieller Werbestrategien besteht gerade darin, dass uns die Gründe für unser Handeln nicht propositional einsichtig gemacht werden sollen. Vorausgegangene Werbeerlebnisse werden in unseren Handlungsentscheidungen überwiegend nicht zum Gegenstand elaborierter Sprachspiele. Dennoch bestehen, wie wir gesehen haben, gute Gründe, ihnen Folge zu leisten. Drittens scheint generell ein menschliches Eigeninteresse darin $\mathrm{zu}$ bestehen, stetig neue Praktiken zu erlernen. Wie Aristoteles bemerkt hat, streben alle Menschen von Natur aus nach Wissen, ${ }^{39} \mathrm{~d}$. h. nach bislang unbekannten Praktiken, mit denen sich Sachverhalte in elaborierter Weise verständlich machen lassen. Einübungsprozesse begegnen uns beispielsweise im Bereich des Sports, des Erlernens fremder Sprachen oder in Weiterbildungsseminaren. Die Einwilligung der Akteure in solche Praktiken kennzeichnet sie als ausgewiesene Bereiche der Einübung. Viertens liefern sportliche Aktivitäten sowie viele andere Bereiche unseres alltäglichen Lebens Beispiele für ein Handeln ohne Überlegen. ${ }^{40}$ Handlungen aus Gewohnheit unterscheiden sich von spielerischem Verhalten auf der Entwicklungsstufe von Kleinkindern dadurch, dass sie darüber hinaus auch auf einer höheren Entwicklungsstufe auftreten. Die anfängliche Einführung in diese Handlungszusammenhänge ist zu diesem Zeitpunkt abgeschlossen, und die routinemäßige Beherrschung führt darüber hinaus mitunter dazu, dass die Akteurin nicht vor jeder Handlung Überlegungen darüber anstellen muss, wie sie die Handlung in der richtigen Weise auszuführen hat. Durch die habitualisierende Einübung wird ein Überlegungsprozess überflüssig.

\section{4}

Innerhalb einer Sprachgemeinschaft teilen wir in vielen Fällen einen gemeinsamen Standpunkt, weil wir in Bezug auf ein Urteil in gleicher Weise in den Gebrauch eines betreffenden Sprachspiels eingeführt worden sind. Ohne diese notwendige Konformität wäre das soziale Leben, wie wir es kennen, nicht vorstellbar, da sie eine Verständigung über gemeinsame Praktiken hinweg in einer Sprachgemeinschaft garantiert. ${ }^{41}$ Die Konformität im Sinne einer diskursiven Übereinstimmung ist demgegenüber ein Spezialfall geteilter Praktiken des sozi-

39 Vgl. Aristoteles (1995), 980a21.

40 Vgl. Kern (2010).

41 PU 242: „Zur Verständigung durch die Sprache gehört nicht nur eine Übereinstimmung in den Definitionen, sondern (so seltsam dies klingen mag) eine Übereinstimmung in den Urteilen.“ 
alen Lebens, wie ich eingangs behauptet habe. Wir haben gesehen, dass das Erreichen einer diskursiven Übereinstimmung im Rahmen elaborierter Sprachspiele eine ganze Reihe an Vorbedingungen stellt. Dennoch neigen wir dazu zu glauben, dass die Möglichkeit der Übereinstimmung durch das Geben und Annehmen von Gründen den Normalfall unter Menschen als rational agierenden Wesen darstelle. Die Überbewertung, die mit dieser Sichtweise verbunden ist, führt zu einem verzerrten Selbstbild des Menschen: Denn der im eigenen Selbstverständnis eingestellte Fokus auf die elaborierten Sprachspiele innerhalb einer Praxis des Überzeugens lässt oftmals die Präsuppositionen primitiver Praktiken außer Acht, deren gemeinsame Verwendungsweise eine Übereinstimmung in den argumentativ angeführten Überzeugungen überhaupt erst möglich macht. Insofern konzentriert sich dieses Selbstbild gewissermaßen lediglich auf die Spitze des Eisbergs.

Wie die Analyse des Konfliktes zwischen zwei in grundlegenden Praktiken verschiedenen Sprachgemeinschaften, den Gruppen A und B, gezeigt hat, besteht der einzige Weg aus dem Dilemma zwischen dem Bestehen grundlegend verschiedener Praktiken und der Forderung, diese Pluralität mit dem Ziel, ein gegenseitiges Verständnis zu erreichen, einzuebnen, im Vorgang der Überredung. An den Stellen, an denen Menschen mit Überzeugungsversuchen an ihre Grenzen stoßen, beginnt ein schleichender Prozess, in dem das anfängliche Überzeugen seinen wahren grundlegenden Charakter des Überredens offenbart. Argumente scheinen, so gesehen, gezielte Instrumente zur Überredung zu sein.

Diese Schlussfolgerung sollte aber nicht zu dem Missverständnis führen, den Vorgang als geeignetes Instrument zur gezielten Erwirkung politischer Macht zu verstehen. Denn zumindest gegenüber Menschen, die bereits im Besitz elaborierter Praktiken sind, bietet die Überredung, auch wenn man sie als ein gezieltes Instrument der Manipulation verstehen möchte, keine Möglichkeit, die Umformung fremder Praktiken, die mit dem manipulativen Eingriff erreicht werden soll, zu kontrollieren. Die Folge einer Überredung ist, wie wir gesehen haben, eine Umformung bestehender Praktiken und damit verbundener Überzeugungen in einem System, das sich nach einer Konfrontation in unkontrollierter und unkontrollierbarer Weise erneut formieren muss. Insofern kann der Ausgang des Versuchs, eine Person zu überreden, nicht auf exakte Weise vorausgesehen werden. Die Beispiele chaotischer Reaktionen, die mit kontrollierten Umformungsversuchen durch manipulative Überredung verbunden sind, gehen über die Grenzen individueller Personen hinaus, und zeigen sich darüber hinaus in bilateralen Beziehungen zwischen Staaten, deren Umformungsversuche sich bis zu kriegerischen Auseinandersetzungen ausbilden können. 
Überredungen stellen Vorgänge eines dynamischen Prozesses dar, in denen ein System von Praktiken durch manipulatives Eingreifen geformt und umgeformt wird. In unserem Anspruch, als rationale Wesen den Ausgang dieser Vorgänge voraussagen zu können, beziehen wir uns oftmals auf Regularitäten, die wir als „rational“ ausweisen. Demgegenüber halten wir den Vorgang des Überredens für eine dem Geben und Annehmen von Gründen entgegenstehende Praxis, die als wenig intellektuell und sogar unredlich angesehen wird. $\mathrm{Zu}$ dieser Sichtweise hat die philosophische Tradition ihren Beitrag geleistet. Es stellt sich jedoch heraus, dass die Überredung den rationalen Praktiken nicht entgegensteht, sondern ihnen gegenüber logisch sogar vorausgesetzt werden muss. Diese Tatsache stellt den Ausgangspunkt einer zukünftigen Neujustierung unseres menschlichen Selbstverständnisses als rationale Wesen dar. ${ }^{42}$

42 Ich danke Britt Harrison und Nigel Pleasants für hilfreiche Anmerkungen zu der englischsprachigen Fassung des Manuskripts.

\section{Literatur}

Aristoteles (1995), Metaphysik, übers. v. Bonitz, H., Hamburg.

Brandom, R. B. (2000), Articulating Reasons. An Introduction to Inferentialism, Cambridge, Mass.

Davidson, D. (1980), Essays on Actions and Events, Oxford.

Diels, H., u. Kranz, W. (Hg.) (1903), Fragmente der Vorsokratiker, Berlin.

Diemer, A. (1972), Fürwahrhalten, in: HWPh 2, 1149-1150.

Forst, R., (2007), Das Recht auf Rechtfertigung, Frankfurt am Main.

Franken, F. (2011), The Concept of Objective Certainty and the Conception of Dynamic Knowledge, in: Jäger, C., u. Löffler, W. (Hg.), Epistemology: Contexts, Values, Disagreement, Papers of the 34th International Wittgenstein Symposium, Kirchberg am Wechsel.

Franken, F. (2013), Making up the Rules as we go along: The Practicability Approach, in: Moyal-Sharrock, D., Munz, V., u. Coliva, A. (Hg.), Mind, Language and Action, Papers of the 36th International Wittgenstein Symposium, Kirchberg am Wechsel.

Franken, F. (2014), Praxis und Gründe. Zu den normativen Grundlagen praktischer Rationalität, Diss., München.

Freud, S. (2007), Totem und Tabu, 10. Aufl., Frankfurt am Main.

Hacker, P. M. S. (2007), Human Nature: The Categorial Framework, Malden, Mass., Oxford.

Hempel, C. G., u. Oppenheim, P. (1948), Studies in the Logic of Explanation, in: Philosophy of Science 15.2, 135-175.

Hertzberg, L. (1988), On the Attitude of Trust, in: Inquiry 31.3, 307-322.

Hume, D. (1978), A Treatise of Human Nature, hg. v. Selby-Bigge, L. A., u. Nidditch, P. H., 2. Aufl., Oxford.

Kant, I. (1983), Kritik der reinen Vernunft, hg. v. Weischedel, W., Darmstadt. 
Kern, A. (2010), „Handeln ohne Überlegen“, in: Tolksdorf, S. (Hg.), In Sprachspiele verstrickt. Oder: Wie man der Fliege den Ausweg zeigt; Verflechtungen von Wissen und Können, Berlin u. New York, 193-220.

Kober, M. (1993), Gewissheit als Norm. Wittgensteins erkenntnistheoretische Untersuchungen in Über Gewissheit, Berlin u. New York.

Korsgaard, C. (1997), The Normativity of Instrumental Reason, in: Cullity, G., u. Gaut, B. (Hg.), Ethics and Practical Reason, Oxford, 215-254.

Mann, W. R. (1992), Rechtfertigung I, in: HWPh 8, 251-256.

McDowell, J. (1995), Might There Be External Reasons?, in: Altham, J. E. J. (Hg.), World, mind, and ethics. Essays on the ethical philosophy of Bernard Williams, Cambridge, 387-398.

Moyal-Sharrock, D. (2007), Understanding Wittgenstein's On Certainty, London.

Platon (2011), Werke, hg. v. Eigler, G., 6. Aufl., Darmstadt 2011 [verwendete Abkürzungen: Gorg. $=$ Gorgias; Phaidr. = Phaidros; Prot. $=$ Protagoras $]$.

Ritter, J., Gründer, K., u. Gabriel, G. (Hg.) (1971 ff.), Historisches Wörterbuch der Philosophie [HWPh], 13 Bde., Darmstadt.

Schneider, H. J. (2002), Beruht das Sprechenkönnen auf einem Sprachwissen?, in: Krämer, S., u. König, E. (Hg.), Gibt es eine Sprache hinter dem Sprechen?, Frankfurt am Main, 129-150.

Stroud, B. (1997): Hume, London.

Tolksdorf, S. (2011), Wittgenstein und das Projekt einer pragmatisch-genealogischen Philosophie der Sprache, in: Majetschak, S. (Hg.), Wittgenstein-Studien 2, 103-136.

Williams, M. (2000), Wittgenstein and Davidson on the Sociality of Language, Journal for the Theory of Social Behaviour 30.3, 299-318.

Williams, M. (2010), Blind Obedience. Paradox and learning in the later Wittgenstein, London.

Wittgenstein, L. (1984a): Bemerkungen über die Philosophie der Psychologie. Letzte Schriften über die Philosophie der Psychologie (= Werkausgabe 7) [BPP], Frankfurt am Main.

Wittgenstein, L. (1984b): Bemerkungen über die Farben. Über Gewißheit. Zettel. Vermischte Bemerkungen (= Werkausgabe 8) [ÜG], Frankfurt am Main.

Wittgenstein, L. (1989), Ursache und Wirkung. Intuitives Erfassen [UW], in: Vortrag über Ethik und andere kleine Schriften, hg. u. übers. v. Schulte, J., Frankfurt am Main.

Wittgenstein, L. (2009), Philosophical Investigations [PU], hg. v. Hacker, P. M. S, u. Schulte, J., 4. Aufl., Oxford. 